\title{
A mathematical model of absorbing Markov chains to understand the routes of metastasis
}

\author{
David H. Margarit ${ }^{* \dagger}$ and Lilia Romanelli $* \dagger$ \\ * Instituto de Ciencias, Universidad Nacional de General Sarmiento, Buenos Aires, Argentina \\ $\dagger$ Consejo Nacional de Investigaciones Científicas y Técnicas (CONICET), Buenos Aires, Argentina \\ dmargari@ungs.edu.ar, lili@ungs.edu.ar
}

Received: 28 December 2015, accepted: 28 July 2016, published: 2 August 2016

\begin{abstract}
Metastasis is a complex and multi-step stochastic process. The study of the probabilities of generating a tumor from a primary site in another organs is the aim of this work. Based on statistics of National Institute of Cancer of Argentina (INC), a characterization of the routes of metastasis for the principal organs is presented by using Absorbing Markov chains. The metastasis propagation from different primary sites towards secondary and tertiary sites is also shown, emphasizing the relation and analysis about absorbing states.
\end{abstract}

Keywords-Metastasis; Complex Systems; Mathematical Modelling; Absorbing Markov chains

\section{INTRODUCTION}

Cells have a specific and stipulated time of death (apoptosis) and reproduction rate to maintain cell balance. A tumor is the result of an uncontrolled growth of abnormal cells or when cells lose the ability to die. These cells form accumulations that affect the normal functioning of the organs, and can spread to other organs so as to cause metastasis. Metastasis is the spread of circulating tumor cells (CTC) from a primary site to near or distant locations by different ways. This depends on the organ and its initial localization through either the bloodstream or the lymphatic system (a collection of vessels that carry fluid and immune cells)[1], [2].

Markov chains are used to model different natural systems based on statistics and applications ([3], [4]), making the dynamic and continuous processes where the states depend only on the actual state and not as a result of previous events[5]. Recently, some works ([6], [7]) give different approaches by using Markov chains in metastasis processes from the lung, where mechanisms of progression and time scales of systemic disease are quantized. In the present work, we use absorbing Markov chains to analyse the metastasis transmission of solid tumors of different organs: from the primary site to a secondary site (called metastasis from primary site), and from there to a tertiary site (metastasis of secondary site) for the principal cancers in Argentina. The probabilities of having a tumor in a tertiary or secondary site from a primary site and their differences depending on each organ is analysed, as well as the probabilities that from a primary site ends in those organs that have very low probability of spreading CTCs are calculated. Finally, the steps (meaning the stages between metastasis from a primary to secondary or tertiary 
site) based in absorbing states (in our case, organs with low probability of generating metastasis) are found. The aim of the present work is to understand how the organs are related to each other giving a characterization of the routes of metastasis.

The metastasis are different for males and females. However the analysis performed here is similar in both cases, although different organs are affected (ovaries, vagina or uterus in females and prostate or testicles in males). In this paper we refer only the metastasis in males as a case study.

This work is organized as follow: In Section II a brief description of the methodology is given; Section III is devoted to describe transition matrices and absorbing states; The transition matrix for tertiary sites is shown in Section IV An analysis of expected number of steps and probability of absorption by absorbing states is found in Section V; Finally, comments and conclusions are drawn in Section VI.

\section{Methodology}

The Markov chain transition matrix $P$ was assembled and it is determined by the number of organs with higher probabilities of developing metastasis. This matrix shows the probability that an organ can be reached by CTCs[ [8] from another organ and has been built under the assumption of developing metastasis. Up to now, three leading routes of metastasis are known: Hematogenous (blood circulation), lymphatic and transcoelomic. With this information, the statistics of National Institute of Cancer of Argentina[9] of the principal tumors and quantitative data on the main organs affected depending on the primary site (obtained from National Cancer Institute at the National Institutes of Health of United States)[10], we performed the graph (depicted in Fig. 1) showing the most common tumors and the principal sites where they can generate metastasis.

The graph was designed by free software Visone 2.15[11]. The size of the nodes represents

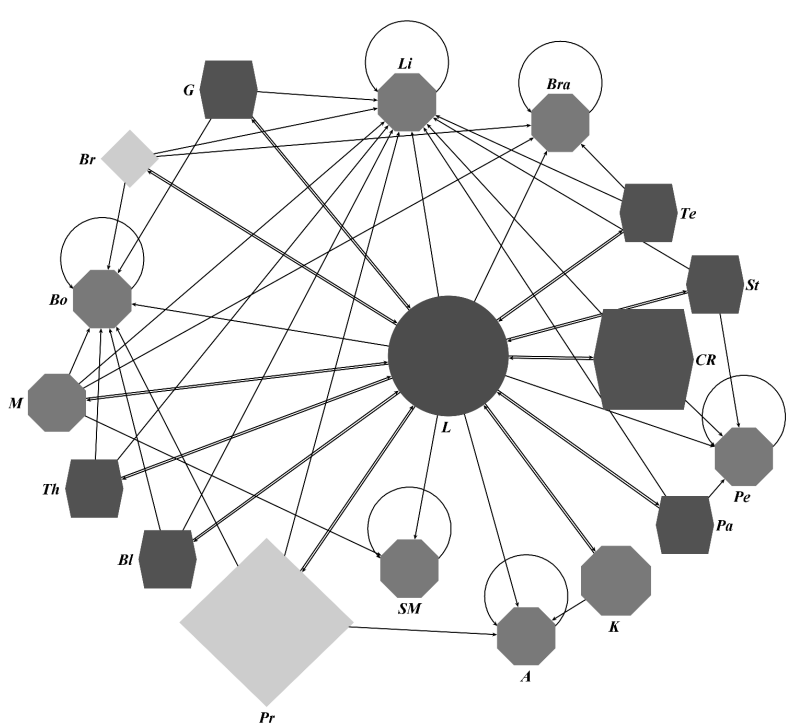

Fig. 1: Graph for the principal links of metastasis for tumors in males based in statistics. The name of the organs were referred by a symbol as depicted in Table I

the proportion of cases for the main tumors mentioned in reference [9]. The connection of these nodes (the links) is based on the main sites of metastasis, for each specific organ, according to the data of the reference [10]. Besides, the shapes of the nodes depends on the amount of linked organs: Circle, 6 or more organs to propagate metastasis; Rhombuses, 4 or more; Hexagons, 2 or more; and finally Octagons, organs with low probability of propagate metastasis. It is important to note that the organs with low probability of generating metastasis, compared to the rest, are shown in the graph as a link on themselves (not to be confused as to metastasize about themselves).

In Fig. 1, it can be seen that the Lung is the principal link. Although the principal primary sites are Prostate, Colon or Rectum, Lung tumor is the most common but usually comes from some primary site, being the principal secondary site ([12], [13]). 
D. H. Margarit et al., A mathematical model of absorbing Markov chains ...

TABLE I: Symbols for organs

\begin{tabular}{|c|c|}
\hline ORGAN & NAME \\
\hline Adrenal Gland & $\mathrm{A}$ \\
Bladder & $\mathrm{Bl}$ \\
Bone & $\mathrm{Bo}$ \\
Brain & $\mathrm{Bra}$ \\
Breast & $\mathrm{Bre}$ \\
Colon/Rectum & $\mathrm{CR}$ \\
Gallbladder & $\mathrm{G}$ \\
Kidney & $\mathrm{K}$ \\
Liver & $\mathrm{Li}$ \\
Lung & $\mathrm{L}$ \\
Melanoma & $\mathrm{M}$ \\
Pancreas & $\mathrm{Pa}$ \\
Peritoneum & $\mathrm{Pe}$ \\
Prostate & $\mathrm{Pr}$ \\
Skin/Muscle & $\mathrm{SM}$ \\
Stomach & $\mathrm{St}$ \\
Testicular & $\mathrm{Te}$ \\
Thyroid & $\mathrm{Th}$ \\
\hline
\end{tabular}

\section{TRANSITION MATRIX, PROBABILITIES AND ABSORBING STATES}

\section{A. Transition matrix and its probabilities}

The characterization of metastatic evolution is developed by using Markov chains, based on a network construction from a primary site.

Let $X_{0}$ (primary site) be the organ where the tumor was originated, and $X_{1}$ the state of the process where the new tumor is formed coming from the primary site and develop metastasis (Note: The transition time, sub index of $X$, does not refer to a calendar time, it refers to a general time in which has already been observed a new tumor). The probability that an organ develops metastasis from another one is:

$$
p_{i j}=P\left[X_{1}=j \mid X_{0}=i\right]
$$

where $i, j=1,2,3 \ldots, m$ number of organs.

The values $p_{i j}$ are called transition probabilities [14] and have two properties:

- $\sum_{j=1}^{m} p_{i j}=p_{i 1}+p_{i 2}+\ldots+p_{i m}=1$, since the system must be in one of these states $m$, the sum of probabilities must be equal to 1 .
This means that the elements in any row of the matrix transition must add 1 .

- Each element $p_{i j} \geq 0$

Based in the transition probabilities, the transition matrix $P$ is given by:

$$
P=\left[p_{i j}\right], \quad P \in \mathbb{R}^{m \times m}
$$

The routes for metastasis from one organ to another are known; although, in the literature, no information is available about their relative likelihood on which organs have an advantage over others. Given this slight uncertainty, the qualitative information ([10], [15]), we assume an equal probability that an organ $X_{0}$ (primary site), reaches other one $X_{1}$ (secondary site), this is under the assumption a metastasis is detected and based in the possibles routes as previously discussed. For other cases, where there are not predominant organs for metastasis from a specific primary site (according references [10], [12] and [13]), we will assume zero probability in order to work only the predominant sites of metastasis.

Looking at matrix $P$, if a tumor of a primary site, for example Prostate (Fig. 3a), has a non null probability of developing a new tumor in a secondary site, this will be $1 / 4$ for $L, L i, B o$ and $P e$. The same considering a tumor in Stomach (3b), that probability will be $1 / 3$ for $L i, P e$ and $L$.

The matrix $P$ is given in the Appendix A. Another way to visualize the matrix $P$ is displayed in the Fig. 2, where, in RGB scale, the probabilities expressed in Eq. 2 can been seen.

\section{B. Absorbing states}

It is worth to notice the existence of absorbing states in the system, these are states where it is impossible to leave and are found if any row of the matrix satisfies[14]:

$$
p_{i i}=1 \text { and } p_{i j} \neq 0 \quad(\text { if } i \neq j)
$$


D. H. Margarit et al., A mathematical model of absorbing Markov chains ...

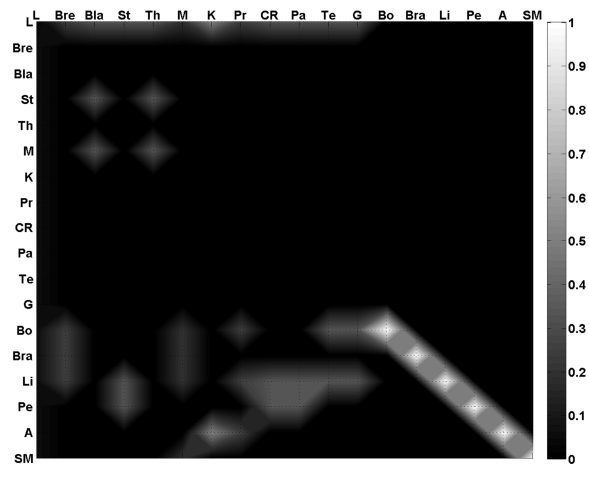

Fig. 2: Visualization of the transition matrix, in gray scale, for secondary sites.

The properties of an Absorbing Markov chain are:

- At least, it has one absorbing state

- The absorption ends in an absorbing state with probability 1

In this work, the transition matrix was performed from the principal organs with higher probability for developing metastasis. However, for some organs, if the tumor is originated there or if it is developed elsewhere (first or secondary sites), do not evolve as metastasis from there. The absorbent states are organs that rarely metastasize, i.e. these are organs do not generate metastasis in the next step of the transition matrix. The Absorbing States of $P$ are: Bone (Bo), Brain (Bra), Liver (Li), Peritoneum (Pe), Adrenal Gland $(A)$ and Skin/Muscle (SM).

\section{TRANSITION MATRIX FOR TERTIARY SITES (SECOND METASTASIS)}

For the process to move from state $i$ to state $j$ in two steps, it must go through an intermediate state $k$. If a tumor in a secondary site spreads to a new organ (tertiary site), this will be labelled as $X_{2}$. The probability of generate a new tumor in a tertiary site from a primary site is given by:

$$
p_{i j}^{(2)}=P\left[X_{2}=j \mid X_{0}=i\right]=\sum_{k=1}^{m} p_{i k} \cdot p_{k j}
$$

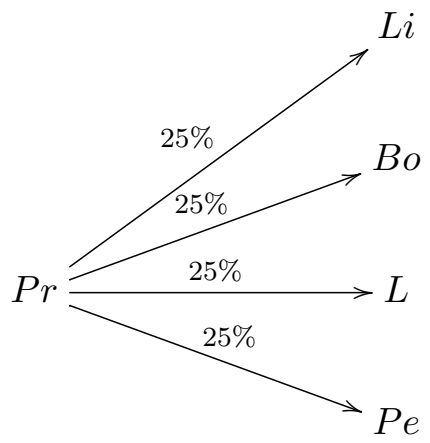

(a)

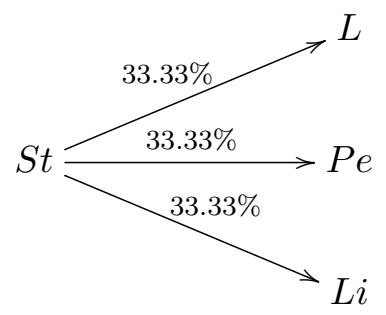

(b)

Fig. 3: Probabilities (in percentage) of metastasis in organs from Prostate (a) and Stomach (b) cancer respectively.

where $i, j, k=1,2,3 \ldots, m$ number of organs. Similarly Eq. 2, the new transition matrix for Second Step $P^{(2)}$ is built for males and this is given by:

$$
P^{(2)}=\left[p_{i j}^{(2)}\right], \quad P^{(2)} \in \mathbb{R}^{m \times m}
$$

Eq. 5 gives information how to obtain a second metastasis from the original tumor going through the possible connections. Although the metastasis from metastasis is unlikely, some clinical evidence was found in cites [16], [17]. The matrix $P^{(2)}$ is shown Appendix B.

Therefore, $P^{(2)}$ allows to find the probabilities of metastasis in a tertiary site from a primary site. For sake of clarity, examples are shown in Fig. $4 a$ and $4 \mathrm{~b}$.

A global analysis of the matrix $P^{(2)}$ shows that if the primary site is the Lung $(L)$, absorbing 


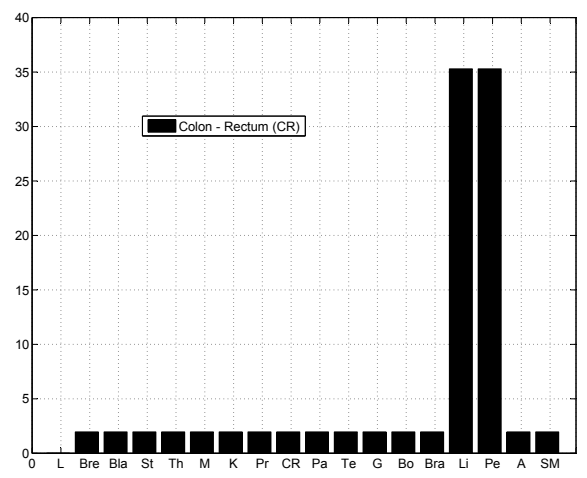

(a)

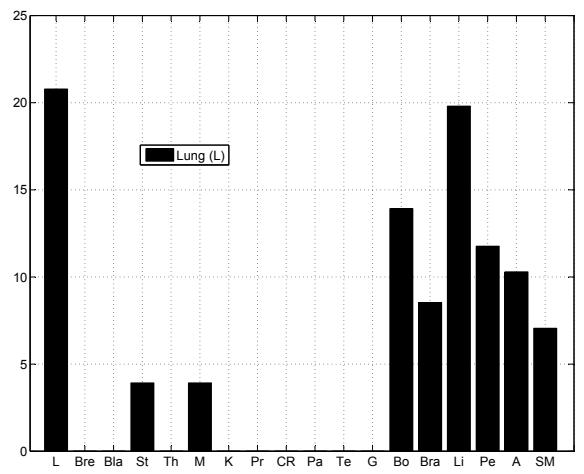

(b)

Fig. 4: Probabilities (in percentage) for tumors in tertiary site from (a) Colon/Rectum $(C R)$ and (b) Lung $(L)$.

states are the most probable tertiary sites as discussed in Sec. 3 (Bo, Bra, Li, Pe,A and $S M)$. This remarks the role of Lung as the principal link between organs[18]. For other organs (disregarding the absorbing states), if the tumor is developed, there is some probability to generate metastasis in a tertiary site.

In sum, according with recent statistics[8], the principal tumors in the population are Lung $(L)$ and Colon/Rectum $(C R)$ tumor. In the $P$ matrix can be observed the probabilities for evolving a tertiary site tumor from those two. If Colon/Rectum tumor is a primary site, the principal tertiary sites are Liver $(\mathrm{Li})$ and Peritoneum $(\mathrm{Pe})$. And, if there is a Lung tumor, the principal tertiary sites are the absorbing states $(B o, B r a, L i, P e, A$ and $S M)$, as it was previously discussed. This can be visualized in the Fig. 5 .

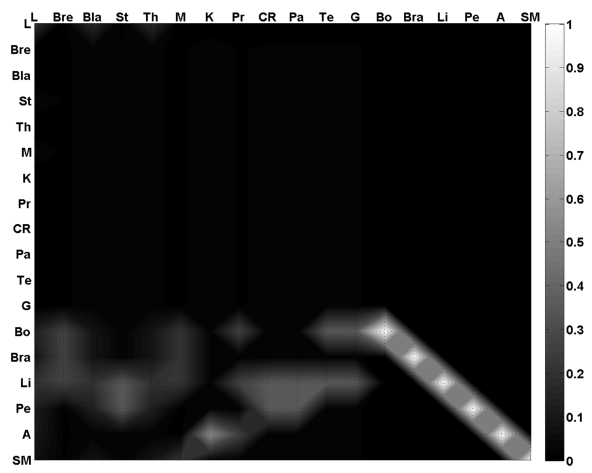

Fig. 5: Visualization of the transition matrix, in gray scale, for tertiary sites.

\section{ANALYSIS OF THE EXPECTED NUMBER OF STEPS AND PROBABILITY OF ABSORPTION BY ABSORBING STATES}

When the processes are absorbing, the number of steps before the system is absorbed, as well as, the probability of absorption of any absorbing state can be found. In order to find this process, each transition matrix will be represented in its canonical form[14], called $J$. It is composed by 4 sub-matrices: $N$ (this sub-matrix contains the probabilities are moving from a non-absorbing state to another non-absorbing state), $A$ (submatrix that contains the probabilities of going from a non-absorbing state to another absorbing state), $O$ (zero sub-matrix) and $I$ (identity sub-matrix).

$$
J=\left(\begin{array}{c|c}
N & A \\
\hline O & I
\end{array}\right)
$$

The matrices with smaller probability contain elements that originate $a$ absorbing states and $n$ non-absorbing states. There are $a+n=m$ states 
D. H. Margarit et al., A mathematical model of absorbing Markov chains ...

of the system.

Let be the fundamental matrix $F=I+N+$ $N^{2}+\cdots=(I-N)^{-1}[14]$. We can calculate from a transient state the expected number of steps before being absorbed by an absorbing state. Let $t_{i}$ be the expected number of steps before the chain is absorbed when this begins in a transient state $i$, and let $\bar{t}$ be the column vector whose $i-t h$ entry is $t_{i}$. Then, the vector $\bar{t}$ can be estimated by the following expression[14]:

$$
\bar{t}=F \cdot \bar{c}
$$

where $\bar{c}$ is vector whose entries are all one.

$\left.\begin{array}{c|c}L & \text { steps } \\ \text { Bre } & 2.237 \\ \text { Bla } & 2.559 \\ S t & 1.745 \\ T h & 2.810 \\ M & 1.447 \\ K & 2.118 \\ P r & 1.559 \\ C R & 1.745 \\ P a & 1.745 \\ T e & 1.745 \\ G & 1.745\end{array}\right]$

\begin{tabular}{|c|c|c|c|c|c|c|}
\hline & Bo & Bra & $\begin{array}{l}Z= \\
L i\end{array}=$ & $P e$ & $A$ & $S M$ \\
\hline$L$ & 0.190 & 0.120 & 0.283 & 0.169 & 0.133 & 0.10 \\
\hline Bre & 0.297 & 0.280 & 0.320 & 0.042 & 0.033 & 0.025 \\
\hline Bla & 0.164 & 0.128 & 0.322 & 0.197 & 0.068 & 0.118 \\
\hline$S t$ & 0.063 & 0.040 & 0.427 & 0.389 & 0.044 & 0.033 \\
\hline$T h$ & 0.164 & 0.128 & 0.322 & 0.197 & 0.068 & 0.118 \\
\hline$M$ & 0.238 & 0.224 & 0.256 & 0.033 & 0.026 & 0.220 \\
\hline$K$ & 0.095 & 0.060 & 0.141 & 0.084 & 0.566 & 0.050 \\
\hline $\operatorname{Pr}$ & 0.297 & 0.030 & 0.320 & 0.042 & 0.283 & 0.025 \\
\hline$C R$ & 0.063 & 0.040 & 0.427 & 0.389 & 0.044 & 0.033 \\
\hline $\mathrm{Pa}$ & 0.063 & 0.040 & 0.427 & 0.389 & 0.044 & 0.033 \\
\hline$T e$ & 0.063 & 0.040 & 0.427 & 0.389 & 0.044 & 0.033 \\
\hline$G$ & 0.063 & 0.040 & 0.427 & 0.389 & 0.044 & 0.033 \\
\hline
\end{tabular}

Here, the matrix $Z$ shows the probability that a specific organ (not belonging to the absorbing states) is absorbed by an absorbing specific organ. If we take as a reference the estimated sum of all elements of each column (i.e., each absorbing state), the main probabilities are $\operatorname{Liver}(\mathrm{Li})$ and Bones $(\mathrm{Bo})$, following by Peritoneum $(\mathrm{Pe})$ and Adrenal Gland $(A)$.

\section{CONCLUSIONS}

In order to search new ways to understand the metastasis process and its interactions among organs sites of possible metastasis, Absorbing Markov chains were used as a mathematical tool to achieve this goal. A characterization of the route of metastasis was developed. The Lung as the main connector between the primary site and the tertiary site, with defined probabilities in emphasized.

The graphs and their connections, in order to develop the transition matrices for the occurrence of tumors, are a good approximation to the reality. These matrices exhibit the connections and the existence of absorbing states in organs with lower probabilities (almost null) to generate metastasis ([19], [20]) in secondary sites, absorbing states represent organs that are not the source of metastasis (sponges as Newton calls in [6]).

In addition, the probability of absorption of any non-absorbing state by any absorbing states can be calculated, and this is given by[14]:

$$
Z=F . A
$$

By the analysis of the expected steps number and the probability of absorption by absorbing states, it is possible to predict the tertiary sites from the secondary sites[22] (or at least estimated 
them). Furthermore, a quantized approximation of the transition matrices of second step $P^{(2)}$ can be obtained. It is a result useful for treatments and therapies given its predictive character. Also, we know that as we get more data on statistics of metastasis, our analysis will be more accurate. This can be through statistical methods or by predominant tumor cells (Stems, progenitors or differentiated) prevailing in each organ (or migrated from other organs such as CTCs). The latter is our immediate study object.

On the other hand, by the properties of Absorbing Markov chains, it was found that in no more than 2 steps (second metastasis in tertiary site) any absorbing states are reached: $B o, B r a, L i, P e, A$ and $S M$ as can be seen in the references [23], [24], [25], [26] and [27]. This also can be seen as a result from the point of view of our model, the main point is the fact that in 3 steps $\left(P^{3}\right)$, we reach an absorbing state with a high probability (see Appendix C), where the sum of probabilities of each row, in the columns of absorbing organs, is near to 1 .

$\left.\begin{array}{ccc}\multicolumn{1}{c}{} & \sum_{a b s=1}^{6} p_{a b s} \\ L & 0.845 \\ B r e & 0.928 \\ B l a & 0.790 \\ S t & 0.905 \\ T h & 0.790 \\ M & 0.943 \\ K & 0.857 \\ P r & 0.928 \\ C R & 0.905 \\ P a & 0.905 \\ T e & 0.905 \\ G & 0.905\end{array}\right]$

Where abs $=\mathrm{Bo}, \mathrm{Bra}, \mathrm{Li}, \mathrm{Pe}, \mathrm{A}$ and SM (The absorbing states).

This analysis is quite similar for females, taking into account the specific organs (Ovaries, Vagina and Uterus).

\section{ACKNOWLEDGEMENTS}

This work is partially supported by PIO 14420140100016CO from CONICET Argentina. The authors want to thanks to Dr Marcela Reale for their valuable contributions.

\section{REFERENCES}

[1] P. Donald et al., Modeling Boundary Conditions for Balanced Proliferation in Metastatic Latency, Clin tumor Res, 19, 5 (2013), 1063-1070.

[2] P. Donald et al., The Dormancy Dilemma: Quiescence versus Balanced Proliferation, Cancer Res., 73, 13 (2013), 3811-3816.

[3] P. Glaus et al., Identifying differentially expressed transcripts from RNA-seq data with biological variation, Bioinformatics, 13 (2010), 1721-1728.

[4] J. Hadfield, S. Nakagawa, General quantitative genetic methods for comparative biology: phylogenies, taxonomies and multitrait models for continuous and categorical characters, Journal of evolutionary biology, 23 (2010), 494-508.

[5] Tierney, Luke, Introduction to general state-space Markov chain theory, Ed. Springer US, 1996.

[6] P. Newton, Spreaders and Sponges Define Metastasis in Lung tumor: A Markov chain Monte Carlo Mathematical Model, Cancer Research, 73 (2013), 2760-2769.

[7] P. Newton, J. Mason, K. Bethel, L.A. Bazhenova, J. Nieva et al., A Stochastic Markov chain Model to Describe Lung tumor Growth and Metastasis. PLoS One, 7, 4 (2012), 1-18.

[8] J. Aguirre-Ghiso, On the theory of tumor self-seeding. implications for metastasis progression in humans, Breast Cancer Research, 12 (2010), 304.

[9] http://www.msal.gov.ar/inc/index.php/acerca-delcancer/estadisticas

[10] http://www.cancer.gov/cancertopics/what-iscancer/metastatic-fact-sheet

[11] http://www.visone.info

[12] D. Loria et al., Tendencia de la mortalidad por cáncer en Argentina, Cuba y Uruguay en un período de 15 años. Rev. Cubana Salud Pública, 36 (2010), 115-125.

[13] C. Navarro et al., Evaluación externa de registros de cáncer de base poblacional: la Guía REDEPICAN para América Latina, Rev Panam Salud Pública, 34 (2013), 336-342.

[14] G. Modica, Poggiolini, A First Course in Probability and Markov chains (United Kindom: John Wiley \& Sons, Ltd), 2013, 187-195

[15] J. Talmadge, I. Fidler, AACR Centennial Series: The Biology of Cancer Metastasis: Historical Perspective. Cancer Research, 70, 14 (2010), 5649-5

[16] Stanleya et al., Clinical Evidence: Metastases can Metastasize, World J Oncol, 3 (2012), 138-141. 
D. H. Margarit et al., A mathematical model of absorbing Markov chains ...

[17] L. Norton, J. Massagu, Is cancer a disease of selfseeding?, Nature Medicine, 12 (2006), 875-878.

[18] S. Jeffrey et al., The Cellular Basis of Site-Specific Tumor Metastasis, N Engl J Med, 322 (1990), 605-612.

[19] S. Duffy, Estimation of mean sojourn time in breast tumor screening using a Markov chain model of both entry to and exit from the preclinical detectable phase, Statistics in Medicine, 14, 14 (1995), 1531-1543.

[20] A. Mazabraud, Metastases (secondary tumors of bone), Pathology of bone tumours, (1998), 381-390.

[21] L. Weiss, K. Haydock, J. Pickren, W. Lane, Organ Vascularity and Metastatic Frequency, Am J Pathol, 101, 1 (1980), 101-114.

[22] E. Nakamura, Secondary tumors of the pancreas: Clinicopathological study of 103 autopsy cases of Japanese patients, Issue Pathology International, 51, 9 (2001), 686-690.
[23] S. Vanharanta, J. Massague, Origins of Metastatic Traits, Tumor Cell, 24, 4 (2013), 410-421.

[24] M. Cummings et al., Metastatic progression of breast tumor: insights from 50 years of autopsies, Tumor Cell, 232, 1 (2013), 23-31.

[25] C. Voskens et al., Impact of Bone and Liver Metastases on Patients with Renal Cell Carcinoma Treated with Targeted Therapy, European Urology, 65, 3 (2014).

[26] C. Gzell, J. Kench, M. Stockler, G. Hruby, Biopsyproven brain metastases from prostate tumor: a series of four cases with review of the literature, International Urology and Nephrology, 45, 3 (2013), 735-742.

[27] J. Xue, G. Peng, J. Yang, Q. Ding, J. Cheng, Predictive factors of brain metastasis in patients with breast tumor, Medical Oncology, (2013), 30-337. 
D. H. Margarit et al., A mathematical model of absorbing Markov chains ...

\section{APPENDIX}

A. Matrix P: Probabilities to generate metastasis in a secondary site from a primary site.

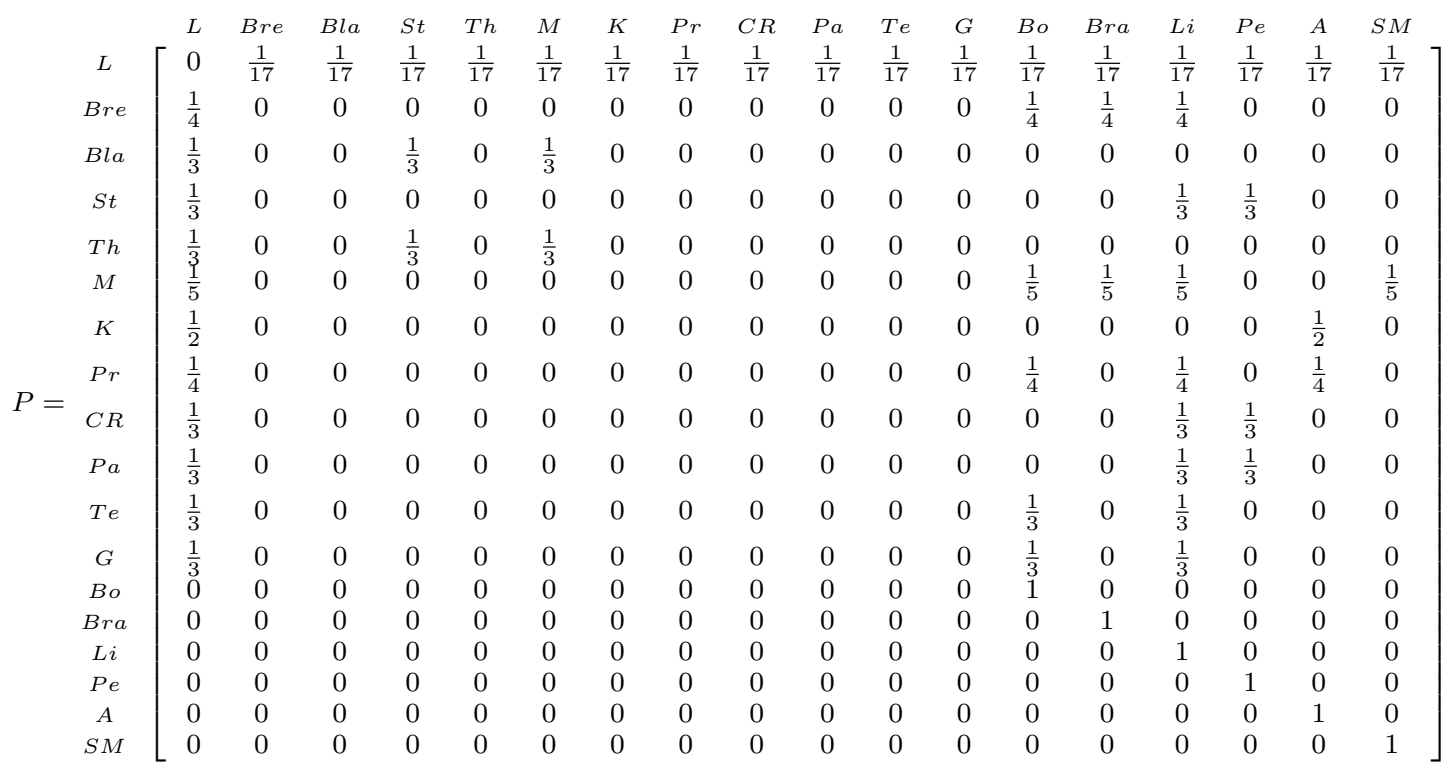

B. Matrix $P^{(2)}$ : Probabilities to generate metastasis in a tertiaryy site from a primary site.

$$
\begin{aligned}
& \begin{array}{llllllllllllllllll}
L & B r e & B l a & S t & T h & M & K & P r & C R & P a & T e & G & B o & B r a & L i & P e & A & S M
\end{array} \\
& \begin{array}{c}
L \\
B r e
\end{array} \quad\left[\begin{array}{ccccccccccccccccccc}
\frac{53}{255} & 0 & 0 & \frac{2}{51} & 0 & \frac{2}{51} & 0 & 0 & 0 & 0 & 0 & 0 & \frac{71}{510} & \frac{29}{340} & \frac{101}{510} & \frac{2}{17} & \frac{7}{68} & \frac{6}{85} \\
0 & \frac{1}{68} & \frac{1}{68} & \frac{1}{68} & \frac{1}{68} & \frac{1}{68} & \frac{1}{68} & \frac{1}{68} & \frac{1}{68} & \frac{1}{68} & \frac{1}{68} & \frac{1}{68} & \frac{9}{34} & \frac{9}{34} & \frac{9}{34} & \frac{1}{68} & \frac{1}{68} & \frac{1}{68}
\end{array}\right]
\end{aligned}
$$

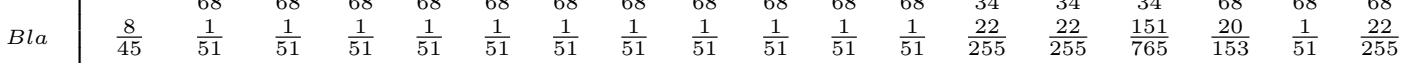

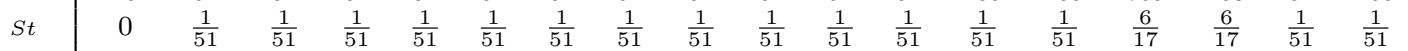

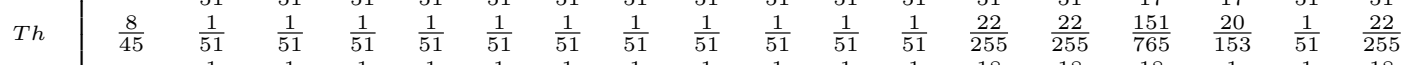

$$
\begin{aligned}
& \begin{array}{lllllllllllllllllll}
M & 0 & \frac{1}{85} & \frac{1}{85} & \frac{1}{85} & \frac{1}{85} & \frac{1}{85} & \frac{1}{85} & \frac{1}{85} & \frac{1}{85} & \frac{1}{85} & \frac{1}{85} & \frac{1}{85} & \frac{18}{85} & \frac{18}{85} & \frac{18}{85} & \frac{1}{85} & \frac{1}{85} & \frac{18}{85}
\end{array} \\
& P^{(2)}=\begin{array}{c|ccccccccccccccccccccc}
K & P r \\
& & 0 & \frac{1}{34} & \frac{1}{34} & \frac{1}{34} & \frac{1}{34} & \frac{1}{34} & \frac{1}{34} & \frac{1}{34} & \frac{1}{34} & \frac{1}{34} & \frac{1}{34} & \frac{1}{34} & \frac{1}{34} & \frac{1}{34} & \frac{1}{34} & \frac{1}{34} & \frac{9}{17} & \frac{1}{34} \\
& & \frac{1}{68} & \frac{1}{68} & \frac{1}{68} & \frac{1}{68} & \frac{1}{68} & \frac{1}{68} & \frac{1}{68} & \frac{1}{68} & \frac{1}{68} & \frac{1}{68} & \frac{1}{68} & \frac{9}{34} & \frac{1}{68} & \frac{9}{34} & \frac{1}{68} & \frac{9}{34} & \frac{1}{68} \\
& 0 & \frac{1}{51} & \frac{1}{51} & \frac{1}{51} & \frac{1}{51} & \frac{1}{51} & \frac{1}{51} & \frac{1}{51} & \frac{1}{51} & \frac{1}{51} & \frac{1}{51} & \frac{1}{51} & \frac{1}{51} & \frac{1}{51} & \frac{6}{17} & \frac{6}{17} & \frac{1}{51} & \frac{1}{51} \\
& 0 & \frac{1}{51} & \frac{1}{51} & \frac{1}{51} & \frac{1}{51} & \frac{1}{51} & \frac{1}{51} & \frac{1}{51} & \frac{1}{51} & \frac{1}{51} & \frac{1}{51} & \frac{1}{51} & \frac{1}{51} & \frac{1}{51} & \frac{6}{17} & \frac{6}{17} & \frac{1}{51} & \frac{1}{51}
\end{array} \\
& \begin{array}{llllllllllllllllllll}
P a & 0 & \frac{1}{51} & \frac{1}{51} & \frac{1}{51} & \frac{1}{51} & \frac{1}{51} & \frac{1}{51} & \frac{1}{51} & \frac{1}{51} & \frac{1}{51} & \frac{1}{51} & \frac{1}{51} & \frac{1}{51} & \frac{1}{51} & \frac{6}{17} & \frac{6}{17} & \frac{1}{51} & \frac{1}{51}
\end{array}
\end{aligned}
$$

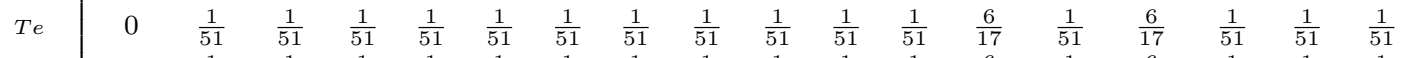

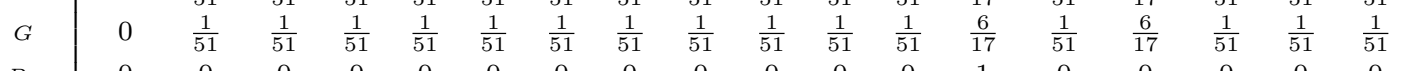

$$
\begin{aligned}
& \begin{array}{l|lllllllllllllllllll}
\text { Bo } & 0 & 0 & 0 & 0 & 0 & 0 & 0 & 0 & 0 & 0 & 0 & 0 & 1 & 0 & 0 & 0 & 0 & 0
\end{array} \\
& \begin{array}{l|llllllllllllllllll}
B r a & 0 & 0 & 0 & 0 & 0 & 0 & 0 & 0 & 0 & 0 & 0 & 0 & 0 & 1 & 0 & 0 & 0 & 0
\end{array} \\
& \begin{array}{lllllllllllllllllll}
L i & 0 & 0 & 0 & 0 & 0 & 0 & 0 & 0 & 0 & 0 & 0 & 0 & 0 & 0 & 1 & 0 & 0 & 0 \\
P e & 0 & 0 & 0 & 0 & 0 & 0 & 0 & 0 & 0 & 0 & 0 & 0 & 0 & 0 & 0 & 1 & 0 & 0
\end{array} \\
& \begin{array}{c|lllllllllllllllllll}
P e & 0 & 0 & 0 & 0 & 0 & 0 & 0 & 0 & 0 & 0 & 0 & 0 & 0 & 0 & 0 & 1 & 0 & 0 \\
A & 0 & 0 & 0 & 0 & 0 & 0 & 0 & 0 & 0 & 0 & 0 & 0 & 0 & 0 & 0 & 0 & 1 & 0 \\
S M & 0 & 0 & 0 & 0 & 0 & 0 & 0 & 0 & 0 & 0 & 0 & 0 & 0 & 0 & 0 & 0 & 0 & 1
\end{array}
\end{aligned}
$$


D. H. Margarit et al., A mathematical model of absorbing Markov chains ...

C. Matrix $P^{(3)}$

\begin{tabular}{|c|c|c|c|c|c|c|c|c|c|c|c|c|c|c|c|c|c|c|}
\hline & $L$ & Bre & Bla & $S t$ & $T h$ & $M$ & $K$ & $P r$ & $C R$ & $P a$ & $T e$ & $G$ & Bo & Bra & $L i$ & $\mathrm{Pe}$ & $A$ & $S M$ \\
\hline$L$ & $\frac{16}{765}$ & $\frac{5}{409}$ & $\frac{5}{409}$ & $\frac{5}{409}$ & $\frac{5}{409}$ & $\frac{5}{409}$ & $\frac{5}{409}$ & $\frac{5}{409}$ & $\frac{5}{409}$ & $\frac{5}{409}$ & $\frac{5}{409}$ & $\frac{5}{409}$ & $\frac{18}{113}$ & $\frac{55}{522}$ & $\frac{43}{186}$ & $\frac{1}{7}$ & $\frac{41}{356}$ & $\frac{33}{364}$ \\
\hline Bre & $\frac{49}{943}$ & 0 & 0 & $\frac{1}{102}$ & 0 & $\frac{1}{102}$ & 0 & 0 & 0 & 0 & 0 & 0 & $\frac{268}{941}$ & $\frac{35}{129}$ & $\frac{183}{611}$ & $\frac{1}{34}$ & $\frac{7}{272}$ & $\frac{3}{170}$ \\
\hline Bla & $\frac{53}{765}$ & $\frac{8}{765}$ & $\frac{8}{765}$ & $\frac{2}{85}$ & $\frac{8}{765}$ & $\frac{2}{85}$ & $\frac{8}{765}$ & $\frac{8}{765}$ & $\frac{8}{765}$ & $\frac{8}{765}$ & $\frac{8}{765}$ & $\frac{8}{765}$ & $\frac{21}{170}$ & $\frac{19}{180}$ & $\frac{15}{59}$ & $\frac{41}{255}$ & $\frac{3}{67}$ & $\frac{77}{765}$ \\
\hline$S t$ & $\frac{53}{765}$ & 0 & 0 & $\frac{2}{153}$ & 0 & $\frac{2}{153}$ & 0 & 0 & 0 & 0 & 0 & 0 & $\frac{20}{431}$ & $\frac{23}{809}$ & $\frac{244}{611}$ & $\frac{19}{51}$ & $\frac{7}{204}$ & $\frac{2}{85}$ \\
\hline$T h$ & $\frac{53}{765}$ & $\frac{8}{765}$ & $\frac{8}{765}$ & $\frac{2}{85}$ & $\frac{8}{765}$ & $\frac{2}{85}$ & $\frac{8}{765}$ & $\frac{8}{765}$ & $\frac{8}{765}$ & $\frac{8}{765}$ & $\frac{8}{765}$ & $\frac{8}{765}$ & $\frac{21}{170}$ & $\frac{19}{180}$ & $\frac{15}{59}$ & $\frac{41}{255}$ & $\frac{3}{67}$ & $\frac{77}{765}$ \\
\hline$M$ & $\frac{18}{433}$ & 0 & 0 & $\frac{2}{255}$ & 0 & $\frac{2}{255}$ & 0 & 0 & 0 & 0 & 0 & 0 & $\frac{18}{79}$ & $\frac{28}{129}$ & $\frac{98}{409}$ & $\frac{2}{85}$ & $\frac{7}{340}$ & $\frac{91}{425}$ \\
\hline$K$ & $\frac{53}{510}$ & 0 & 0 & $\frac{1}{51}$ & 0 & $\frac{1}{51}$ & 0 & 0 & 0 & 0 & 0 & 0 & $\frac{41}{589}$ & $\frac{29}{680}$ & $\frac{91}{919}$ & $\frac{1}{17}$ & $\frac{75}{136}$ & $\frac{3}{85}$ \\
\hline$P r$ & $\frac{49}{943}$ & 0 & 0 & $\frac{1}{102}$ & 0 & $\frac{1}{102}$ & 0 & 0 & 0 & 0 & 0 & 0 & $\frac{268}{941}$ & $\frac{10}{469}$ & $\frac{183}{611}$ & $\frac{1}{34}$ & $\frac{75}{272}$ & $\frac{3}{170}$ \\
\hline$C R$ & $\frac{53}{765}$ & 0 & 0 & $\frac{2}{153}$ & 0 & $\frac{2}{153}$ & 0 & 0 & 0 & 0 & 0 & 0 & $\frac{20}{43}$ & $\frac{23}{80}$ & $\frac{244}{611}$ & $\frac{19}{51}$ & $\frac{7}{204}$ & $\frac{2}{85}$ \\
\hline $\mathrm{Pa}$ & $\frac{53}{765}$ & 0 & 0 & $\frac{2}{153}$ & 0 & $\frac{2}{153}$ & 0 & 0 & 0 & 0 & 0 & 0 & $\frac{20}{431}$ & $\frac{23}{809}$ & $\frac{244}{611}$ & $\frac{19}{51}$ & $\frac{7}{204}$ & $\frac{2}{85}$ \\
\hline$T e$ & $\frac{53}{765}$ & 0 & 0 & $\frac{2}{153}$ & 0 & $\frac{2}{153}$ & 0 & 0 & 0 & 0 & 0 & 0 & $\frac{20}{431}$ & $\frac{23}{809}$ & $\frac{244}{611}$ & $\frac{19}{51}$ & $\frac{7}{204}$ & $\frac{2}{85}$ \\
\hline$G$ & $\frac{53}{765}$ & 0 & 0 & $\frac{2}{153}$ & 0 & $\frac{2}{153}$ & 0 & 0 & 0 & 0 & 0 & 0 & $\frac{20}{431}$ & $\frac{23}{809}$ & $\frac{244}{611}$ & $\frac{19}{51}$ & $\frac{7}{204}$ & $\frac{2}{85}$ \\
\hline Bo & 0 & 0 & 0 & 0 & 0 & 0 & 0 & 0 & 0 & 0 & 0 & 0 & 1 & 0 & 0 & 0 & 0 & 0 \\
\hline $\mathrm{Bra}$ & 0 & 0 & 0 & 0 & 0 & 0 & 0 & 0 & 0 & 0 & 0 & O & 0 & 1 & 0 & 0 & 0 & 0 \\
\hline$L i$ & 0 & 0 & 0 & 0 & 0 & 0 & 0 & 0 & 0 & 0 & 0 & 0 & 0 & 0 & 1 & 0 & 0 & 0 \\
\hline$P e$ & 0 & 0 & 0 & 0 & 0 & 0 & 0 & 0 & 0 & 0 & 0 & 0 & 0 & 0 & 0 & 1 & 0 & 0 \\
\hline$A$ & 0 & 0 & 0 & 0 & 0 & 0 & 0 & 0 & 0 & 0 & 0 & 0 & 0 & 0 & 0 & 0 & 1 & 0 \\
\hline$S M$ & 0 & 0 & 0 & 0 & 0 & 0 & 0 & 0 & 0 & 0 & 0 & 0 & 0 & 0 & 0 & 0 & 0 & 1 \\
\hline
\end{tabular}

